\title{
Effects of geometrical specification of microengineered surfaces to Casimir-Polder potential
}

\author{
A.F Khaidir $^{1 *}$, S.N. Sabki ${ }^{2}$, and M.N.A. Halif ${ }^{1}$ \\ ${ }^{1}$ Institute of Engineering Mathematics, Universiti Malaysia Perlis (UniMAP), Pauh Putra Campus, 02600, Arau, Perlis \\ ${ }^{2}$ School of Microelectronic Engineering, Universiti Malaysia Perlis (UniMAP), Pauh Putra Campus, 02600, Arau, Perlis
}

\begin{abstract}
Cold atoms can be trapped and guided using nanofabricated wires on microengineered surfaces, achieving the scales required by quantum information proposals on the consequences of Casimir-Polder (CP) force to its stability and lifetime. The atoms are found to be less attractive to the thin films compared to dielectric half plate, where the attractive potential drops even further when the thickness of the surface is lower than both thermal wavelength and the spacing length. Amiably, for the spherical surface, the CP potential decays even further as radial thickness decreases. We approximate the CP potential using the analytical expression obtained from the pairwise summation (PWS) calculated by taking a sum of single atom with the jumbled atoms inside the surface, treating them as two-bodies system as a whole. The means of PWS are to calculate the possibilities of geometrical effect: planar and curved surface on the CP potential.
\end{abstract}

\section{Introduction}

The implementation of such nanofabricated wires (atom chips) implanted in microengineered surface over a coherent matter waves of cold atoms for the purpose of trapping and manipulating near the micron distance away from the surface has been underway generating interest to facilitate study of the attractive power of law's effect over the stability and the lifetime of Bose-Einstein Condensate (BEC) atoms [1-3]. The transition from various laser cooling techniques, spatially-varying magnetic trap, evaporative cooling to the placement of microelectronic chip near the 'atomic conveyer belt' or atom chips has seen the new challenges to nanotechnological developments during the trapping process of BEC atoms [4]. Apparently, the precise control of the delivery of atoms during the microtrap process is also necessary to minimize further inevitable noise and decoherence leading into improvisation of atom chips in recent years includes an integrated coherent matter wave circuits and a two-photon transition integrated atom chips for deterministic positioning of the atom cloud [5]. The atom chip has been used recently to probe quantum zero dynamics [6] and to prepare any arbitrary family of internal states of rubidium $\left({ }^{87} \mathrm{Rb}\right) \mathrm{BEC}$ atoms yielded in atom-chip-based microtrap in order to increase the lifetime of coherence phase-space densities [7].

Nonetheless, the geometry specification of designated atom chips also needs to be taken in consideration. This is because the geometrical surface has shown greater possibilities in affecting the attractive potential between the two closed dielectric surfaces, none other than the Casimir-Polder $(\mathrm{CP})$ potential which is studied over the years $[8,9]$. CP potential derives from the Casimir's effect's between the two-body systems [10] because of the occurring quantum electromagnetic dipole fluctuations [11] that involves the evanescence waves from the reproduction and annihilation of virtual photons surrounding it [12]. The simulation of this CP potential is shown intuitively on the reflective patterns of atom clouds notably the dynamical excitations and formation of vortex rings when they loaded closer to the surface, probing the possibilities of trapping the micronatom-surface-distance through quantum reflection $[13,14]$. Under a very tight confining magnetic field, the $\mathrm{CP}$ potential overwhelms the evaporative cooling process causing trapping instabilities as many coherent BEC atoms escape during the process [15]. Therefore, coherency cannot be maintained for obtaining pure condensate of cold atoms trapped near microengineered surface of atom chips. Tuning the CP potential as good as maintaining coherency serves as a part of problemsolving to the main goal of this research, this is by specifying the type of geometrical surface that may weaken the CP potential.

\section{A pairwise summation (PWS) method to estimate Casimir-Polder (CP) potential}

Multiple attempts are done to compute the $\mathrm{CP}$ potential either numerically or by analytical method. The computation of $\mathrm{CP}$ potential using conventional exact scattering method is daunted challenging as the $\mathfrak{R}$ operators of the involved bodies need to be assessed first, and there is no known method to find the $\mathfrak{R}$ operator for material surfaces of arbitrary shape [16]. Therefore, any approximation tool like the one we introduced here, a pairwise summation method (PWS) [17] is preferable due to its functionality to choose different geometrical surface at any best without going through the trouble like finding the complex matrix operators. The PWS tool is noted for underestimating the lateral atom-surfaces forces as it covers most of the

\footnotetext{
* Corresponding author: dauswq@gmail.com
} 
geometric corrections for the calculation of dispersive force between the ground state atoms and corrugated surface [18]. The idea of PWS is to sum up the interactions between a single atom and every atom inside the surface, where the single atom is treated as giant coherence single matter waves in ground state energy level interacting with the surface with dielectric permittivity. The PWS method ignores the Van der Waals (vdW) many-body interactions inside the surface because they are negligible compared to the interaction of approaching atom with atoms made up the surface. Using PWS method, the atom-surface interaction can be expressed as volume integral of an atom over all atoms building up the surface,

$$
U_{m}(\vec{r})=-n \int \frac{C_{m}}{|\overrightarrow{|r|}|^{m}} d^{3} \vec{r}
$$

where $d^{3} \vec{r}$ is a volume element of the surface, $n$ is a density of atoms that made up the surface and $C_{m}$ is a interaction coefficient in which $\mathrm{m}=6,7$ defined for nonretarded London-dispersion potential and retarded CP potential, respectively. Also, their atom-atom interaction constants may be written as follow [19],

$$
\begin{aligned}
C_{6} & =\frac{3 \hbar}{16 \pi^{3} \varepsilon_{0}^{2}} \int_{0}^{\infty} \alpha_{\text {atom }}(i \omega) \alpha_{\text {surface }}(i \omega) d \omega \\
C_{7} & =\frac{23 \hbar c}{64 \pi^{3} \varepsilon_{0}^{2}} \alpha_{\text {atom }}(0) \alpha_{\text {surface }}(0)
\end{aligned}
$$

where $\alpha$ are the polarizabilities of respective dielectric bodies, $\omega$ is a mode defined in imaginary term, $i, \hbar$ is Plank constant divided by $2 \pi$ and $\varepsilon_{0}$ is a vacuum permittivity. In this research, we study the interaction of ${ }^{87} \mathrm{Rb}$-atoms with silicon-based[19],

Table 1. Values of $C_{6}$ and $C_{7}$ coeeficients for $\mathrm{a}{ }^{87} \mathrm{Rb}$ atom in front of silicon bodies.

\begin{tabular}{|c|c|}
\hline$C_{m}$ & $\mathrm{Si}$ \\
\hline$C_{6}\left(10^{-48} \mathrm{~J} \mathrm{~m}^{6}\right)$ & 3.23 \\
\hline$C_{7}\left(10^{-55} \mathrm{~J} \mathrm{~m}^{7}\right)$ & 5.12 \\
\hline
\end{tabular}

As both the outer atoms and its nearby surfaces holding atoms together are treated as single macroscopic bodies, their densities, $\mathrm{n}$ are valid enough to be taken into account and related with the surface's permittivity, $\varepsilon$ by Clausius-Mossotti's relations [19],

$$
\frac{n \alpha(\omega)}{3 \varepsilon_{0}}=\frac{\varepsilon(\omega)-1}{\varepsilon(\omega)+2}
$$

Lastly, the total estimated $\mathrm{CP}$ potential, $U_{p}(z)$ can be obtained using simple proposition below [20],

$$
U_{p}(z)=\left(U_{6}^{-1}(z)+U_{7}^{-1}(z)\right)^{-1}
$$

where $\mathrm{m}=6,7$ defined for non-retarded and retarded potential respectively. In the following section, we will apply this Eqn. (5) to compute interaction between an atom and a flat or planar surface: finite (half-plate) and infinite thickness (half-space), and also a spherically curved surface as indicated in Fig. 1.

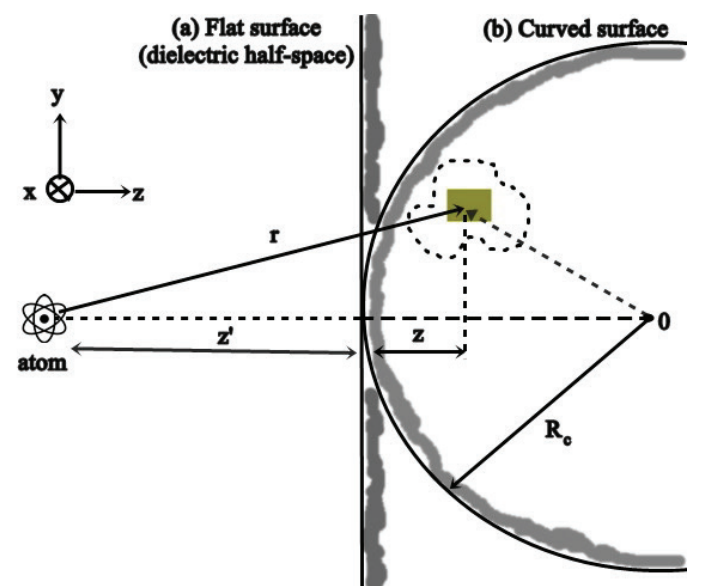

Fig. 1. The illustrations of PWS of approaching atom with (a) a planar surface, and (b) curved surfaces: spherical and cylindrical surface with length, $L$ in $x$-axis. The $z$ ' is longitudal interspace distance while $\mathrm{z}$ is defined as the thickness length for (a) a planar surface, or as radial length for (b) a curved surface with a maximal $R_{c}$. As the atom are brought away from the surface for $z^{\prime}>$ z, atom's perception of curvature gradually changes to flat conception, we will expect the CP potential of curved surface at larger distance are approximately closer to the one calculated for a planar surface. These probe the capability of geometrical surface in affecting the $\mathrm{CP}$ potential.

\subsection{Atom with nearby planar surface}

Let us begin with the application of PWS method to simple case of an infinite planar surface, or dielectric half-space. This means a surface having dielectric permitivity, $\varepsilon(\omega)$ that occupy a half-space of coordinates, defined as $z<0$. Let make assumption that atom is located at $\left(0,0, z+z^{\prime}\right)$, disregarding the $x$ and $y$ coordinates due to translational symmetry. We use the cylindrical coordinates to transform PWS computation of $\mathrm{CP}$ potential into much simpler expressions,

$$
U_{m}(z)=-2 \pi C_{m} \int_{0}^{z} d z^{\prime} \int_{0}^{\infty} \frac{\rho d \rho}{\left[\left(z+z^{\prime}\right)^{2}+\rho^{2}\right]^{\frac{m}{2}}}
$$

where $\mathrm{m}=6,7$. For limit $\mathrm{z} \rightarrow \infty$, the Eqn. (6) becomes the dielectric half-space. While for any arbitrary $z$, the potential is computed for different thickness suited for dielectric semi half-plate, or thin film. Eqn. (6) was to be compared with the one computed using exact scattering method [21],

$$
U_{m \prime}(z)=-\frac{C_{m \prime}}{z^{m^{\prime}}}
$$

where $C_{3}$ and $C_{4}$ are both coefficients calculated from the exact scattering method, canonically different from interaction coefficients we have defined before. Both $U_{3}(z)$ and $U_{4}(z)$ from Eqn. (7) are the Londondispersion potential (non-retarded) and CP potential (retarded) respectively, serve as comparisons to $U_{6}(z)$ and $U_{7}(z)$ obtained by PWS method in Eqn. (6). After 
combining PWS for both regimes using Eqn. (5), the crossover between them, where it can be determined by the effective transition wavelength $\lambda$, are obtained through the coefficients containing the polarizabilities,

$$
\lambda=\frac{3 C_{3}}{4 C_{4}}=\frac{3 C_{7}}{4 C_{6}}
$$

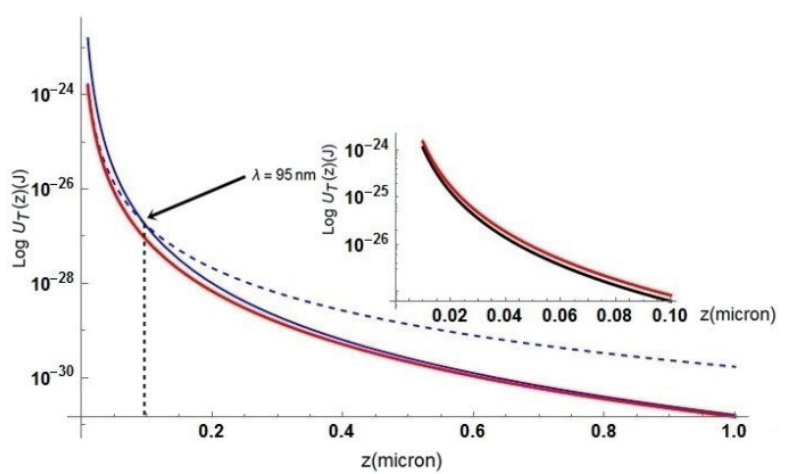

Fig. 2. The top-right graph shows the both attractive potentials of ${ }^{87} \mathrm{Rb}$-atoms decays with the increasing distance from the silicon surface which are computed using PWS method (red color) and exact scattering method (black color) respectively. The bottom graph shows the three different potentials: the nonretardation potential $U_{3}(z)$ represents the short-ranged interaction (blue-dashed), retardation potential $U_{4}(z)$ represents the long-ranged interaction (blue) and the total pairwise summation (PWS) is calculated based on Eqn. (5) (red-bolded). The effective transition wavelength, $\lambda$ was obtained through the intersection point between the nonretardation potential and retardation potential, and was compared with exact scattering method in Eqn. (8).

It is verified in Fig. 2 that $\lambda$ obtained via PWS method is $95 \mathrm{~nm}$, just $5 \%$ error compared to the one calculated from exact scattering method which is $\lambda=$ $100 \mathrm{~nm}$ [21]. By this initial result, the PWS method is still good approximation tool at least for determining the atom's behavior when it approaches different geometrical surface. The error is probably caused by ignoring the $\mathrm{CP}$ potential generated through the interatomic interaction inside the surface. Later, we extend to the different case of thickness, a slab of finite thickness (half-plate). The PWS for this case are formulated as follows,

$$
U_{H P}(z)=U_{P}(z 0+z)-U_{P}(z)
$$

for any $z \geq \lambda$,

$$
U_{H P}(z)=C_{m=3,4}^{\prime}\left\{\frac{1}{(z+z 0)^{m}}-\frac{1}{z^{m}}\right\}
$$

for any $d<z<\lambda$,

$$
U_{H P}(z) \approx-C_{m=3,4}^{\prime} \frac{m d}{z^{m+1}}
$$

where $C_{3}$ a any $z \ll d$ (using the Taylor Series over the predecessor Eqn. 10) where $z_{0}$ is the thickness of the dielectric half-plate. $m=3,4$ refers to $C^{\prime}{ }_{3}$ and $C^{\prime}{ }_{4}$, respectively. This shows that as the thickness $d<<z$, the inverse power-law reduces which cause the nonretardation $U_{3 ; F} \propto-\frac{1}{z^{4}}$ and retardation $U_{4 ; F} \propto-\frac{1}{z^{5}}$ as compares with those thickness when approaching the half-plate. The behavioral change of $\mathrm{CP}$ potential with regards to the slab thickness is shown in Fig. 3.

The inverse power-law reduction has deviated the CP potential of thickness, $d=1 \mathrm{~nm}$ so much from its

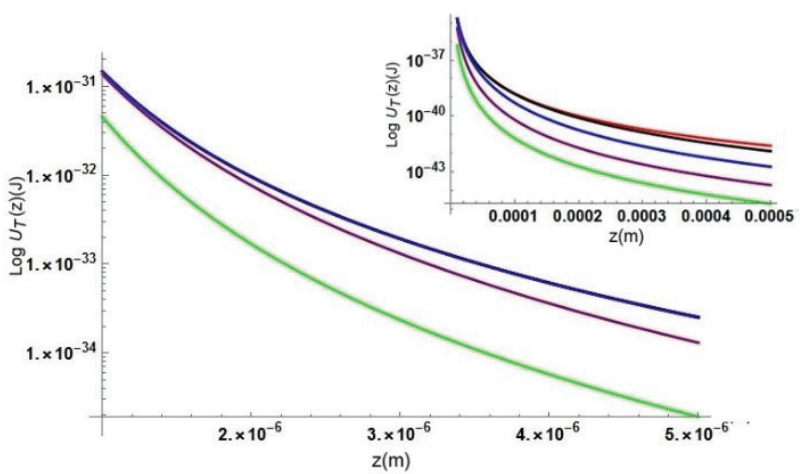

Fig. 3. Comparison of total PWS potentials for each thickness, d: $100 \mu \mathrm{m}$ (black) ,10 $\mu \mathrm{m}$ (blue), 100nm (purple) and $1 \mathrm{~nm}$ (green) for the interaction of ${ }^{87} \mathrm{Rb}$-atoms with $\mathrm{Si}$ thin slabs.

neighboring $d=100 \mathrm{~nm}$ for extremely smaller scale of $z$. From here, it also can be concluded that as thin films become much thinner, CP Potential decays subsequently. But, as d become larger, it almost approaches with the one of half-plate (red color) in Figure 3. These results agree with actual CP potential calculated for thin slabs of dielectric half-plate [22].

\subsection{Atom with nearby spherical surface}

We now focus on the case of spherical surface to which we study how the curvature play a part in shaping CP potential critically as compared with planar one. The PWS for this case is given as follow,

$$
U_{m}\left(z^{\prime}\right)=\pi C_{m} \int_{z^{\prime}-R}^{z^{\prime}+R} \frac{R^{2}-\left(r-z^{\prime}\right)^{2}}{r^{m-1}} d r
$$

where $\mathrm{R}$ is a radius of curvature and $z=z^{\prime}+R$ by referring to Fig.1.

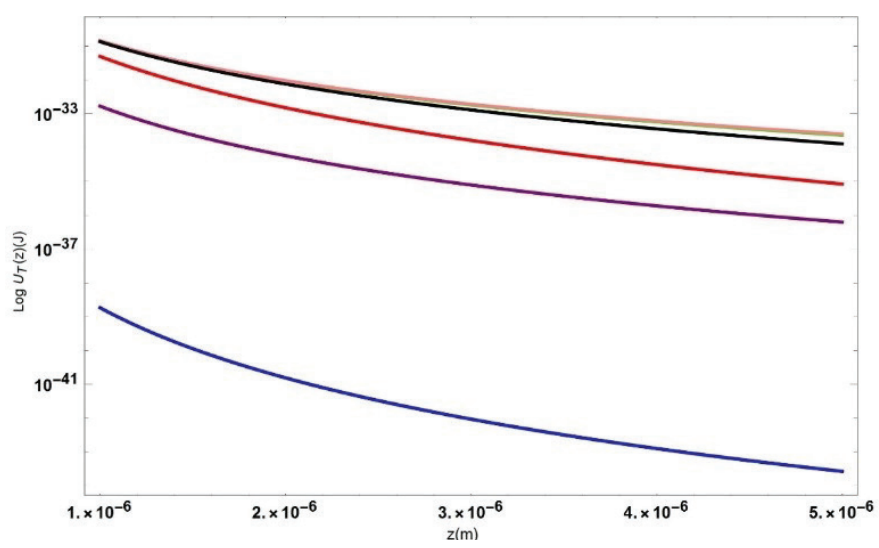

Fig. 4. Comparison of total pairwise potentials $\left({ }^{87} \mathrm{Rb}\right.$-atoms and silicon surface) for both spherical surfaces (curved) and the planar surface (thin slabs) made up of $100 \mu \mathrm{m}$ (green, pink) ,1 $\mu \mathrm{m}$ (red, black) and $1 \mathrm{~nm}$ (blue, purple) thickness respectively. A thin film with thickness, $d=1 \mathrm{~nm}$ doesn't reduce the $\mathrm{CP}$ potential as much as a spherical surface does with similar $R_{S}=1 \mathrm{~nm}$. 
As we may observe in Fig. 4, the spherical surface serves as the best criteria for lowering the $\mathrm{CP}$ potential when compared with planar surface. Coincidently, both spherical and planar surfaces agree that as their thickness increase, they behave like a dielectric half-plate.

\section{Discussions}

Based on the CP potential obtained for the interaction of an atom with the thin slabs of varied thickness and an atom with spherical surface, it clearly shows that geometrical surfaces play vital roles in determining the attractive $\mathrm{CP}$ potential. When interpreting $\mathrm{CP}$ potential of atom-surface interaction, atom's perspective towards the nearby surfaces has eventually changed to dielectric half-space perfection as they are located far away from the source, proven for both case of planar and spherical surfaces. Reducing the attractive CP potential by making the silicon slabs much thinner will help to increase the

\section{References}

1. R. Folman, P. Krüger, D. Cassettari, B. Hessmo, T. Maier, and J. Schmiedmayer Phys. Rev. Lett. 84, 4749 (2000)

2. J. Reichel. Applied Physics B, 74(6):469-487 (2002)

3. M. N. A. Halif, R. Messina and T. M. Fromhold. J. Phys. Conf. Ser. 286012045 (2011)

4. Hansel, W; Hommelhoff, P; Hansch, T W; Reichel, J. Nature; London 413.6855 (2001)

5. R. Roy. An integrated atom chip for the detection and manipulation of cold atoms using a two-photon transition. A thesis submitted to National University of Singapore (2015)

6. S. Gleyzes, and J.M. Raimond. Comptes Rendus Physique 17:7 (2016)

7. C. Lovecchio, F. Schäfer, S. Cherukattil, M. Alì Khan, I. Herrera, F. S. Cataliotti, T. Calarco, S. Montangero, and F. Caruso. Phys. Rev. A 93, 010304(R) (2016)

8. Salem R, Y Japha, J Chabé, B Hadad, M Keil, K A Milton and R Folman. New J. Phys. 12 023039 (2010)

9. M. Keil, O. Amit, S. Zhou, D. Groswasser, Y. Japha, and R. Folman. Journal of Modern Optics, 1-46 (2016) stability and lifetime of $87 \mathrm{Rb}$-atoms trapped nearby the microengineered surface.

The next work we wish to extend to a cylindrical surface: infinitely long cylinder and finite length, $L$. The first case involves the CP computation of infinitely long cylindrical surface with varied thickness, $R_{c}$. The second case attributed to the limit of radial thickness, as $R_{c} \ll z$, whether the close like CP behavior may be observed as in dielectric half-plate (thin films). Lastly, the third case that we will consider when we calibrate the finite length, $L$ cylindrical surfaces. The shrinking of $L$ will change the topological structure of cylindrical surface into a disk, as compared to the affined sphere-like surface. The studies of curved surfaces' effects over CP-potential will fuel up our interests to manipulate atoms over nanofabricated wires forming the microengineered surface (atom chips) with the best condition that it may achieve peculiarly in BEC experiment.

10. H. B. G. Casimir and D. Polder, Phys. Rev. 73, 360 (1948)

11. D. Meschede, W. Jhe, and E. A. Hinds, Phys. Rev. A 41, 1587 (1990)

12. C. Eberlein and S. T. Wu. Phys. Rev. A 68, 033813 (2003)

13. T. A. Pasquini, Y Shin, C. Sanner, M. Saba, A. Shirotzek, D. E. Pritchard, and W. Ketterle, Phys. Rev. Lett. 93, 223201 (2004)

14. R. G. Scott, A. M. Martin, T. M. Fromhold, and F. W. Sheard, Phys. Rev. Lett. 95, 073201 (2005)

15. Y. J. Lin, I. Teper, C. Chin, and V. Vuletić. Phys. Rev. Lett. 92, 050404 (2004)

16. G. Bimonte Phys. Rev. D 95, 065004 (2017)

17. H. Hamaker. Physica 4, 1058 (1937)

18. R. Messina, D. A. R. Dalvit, P. A. Maia Neto, A. Lambrecht, and S. Reynaud, Phys. Rev. A 80, 022119 (2009)

19. R. Messina, M. N. A. Halif, B. Kaczmarek, T. M. Fromhold (Unpublished)

20. F. Shimizu, Phys. Rev. Lett. 86, 987 (2001)

21. S. T. Wu and C. Eberlein. Proc. R. Soc. Lond. A, 455 (1999)

22. A. M. C. Reyes and C. Eberlein. Phys. Rev. A 80, 032901 (2009) 